\title{
Studying halo nuclei by the ratio technique
}

\section{P. CAPEL*}

Physique Nuclaire et Physique Quantique (CP 229)

Universit Libre de Bruxelles (ULB)

B-1150 Brussels, Belgium

E-mail: pierre.capelduLb.ac.be

\section{R. C. Johnson}

Department of Physics

University of Surrey

Guildford GU2 7XH, United Kingdom

\section{F. M. Nunes}

National Superconducting Cyclotron Laboratory and Department of Physics and Astronomy Michigan State University

East Lansing, MI 48824, USA

50th International Winter Meeting on Nuclear Physics - Bormio2012,

23-27 January 2012

Bormio, Italy

\footnotetext{
* Speaker.
} 


\section{Introduction}

The development of radioactive ion beams (RIBs) in the mid 80s, has enabled nuclear physicists to explore the nuclear landscape far away from stability. This technological breakthrough led to the discovery of exotic nuclear structures such as halo nuclei [四]. Halo nuclei exhibit a large matter radius in comparison with their isobars. This peculiarity is qualitatively understood as due to their small separation energy for one or two neutrons [ [ $]$. When these valence neutrons are in a low orbital angular momentum shell, they exhibit a significant probability of presence at a large distance from the other nucleons and hence form a sort of halo around the core of the nucleus [3]].

Archetypes of one-neutron halo nuclei are ${ }^{11} \mathrm{Be}$ and ${ }^{15} \mathrm{C}$, in which the loosely-bound valence neutron is primarily in the $1 s_{1 / 2}$ orbital. In addition to their halo nature, two-neutron halo nuclei exhibit the Borromean property, i.e. whereas the three-body system (core-n-n) is bound none of the two-body subsystems (core-n and n-n) is. Examples of such nuclei are ${ }^{6} \mathrm{He}$ and ${ }^{11} \mathrm{Li}$.

Due to their very short lifetime these nuclei cannot be investigated through usual spectroscopic techniques and information about their structure is inferred from indirect measurements. Breakup [四, 回], knockout [圆], or elastic scattering [ [ $]$ ] have been used to study halo nuclei. However, in order to extract valuable structure information from reaction cross sections, a good understanding of the reaction mechanism is needed. For that reason many models have been developed to analyse reaction measurements (see, e.g., Refs. [ [8, Q] for a review).

Alternatively, one could seek observables independent of the reaction process. We suggest here to take the ratio of angular distributions for breakup and elastic scattering to significantly reduce the sensitivity to the reaction mechanism. This ratio technique is shown to emphasise the information on nuclear structure that can be inferred from reaction measurements [ए]].

These proceedings are structured as follows. In a first step, we show that angular distributions for elastic-scattering and breakup exhibit similar features, i.e. that the projectile is scattered similarly whether it remains bound or is broken up [W]. We then introduce the Recoil Excitation and Breakup (REB) model, which nicely explains this result [[2] and which suggests that ratios of angular distributions contain information only about the structure of the projectile. In Sec. 目, the ratio technique is presented in more details and tested within the Dynamical Eikonal Approximation (DEA) [12], [4]]. The sensitivity of the ratio to the projectile structure is then investigated before summarising in Sec. [1.

\section{Angular distributions for elastic scattering and breakup}

A recent analysis has shown that angular distributions for elastic scattering and breakup of loosely-bound nuclei, such as halo nuclei, exhibit similar features [W]. This is illustrated in Fig. W, where both distributions are plotted for ${ }^{11} \mathrm{Be}$ impinging on $\mathrm{Pb}$ at $69 \mathrm{AMeV}$. The elastic scattering cross section (blue dotted line) is plotted as a ratio to the Rutherford cross section as a function of the scattering angle $\theta$. The breakup cross section is plotted for a ${ }^{10} \mathrm{Be}-\mathrm{n}$ relative energy $E=$ $0.3 \mathrm{MeV}$ as a function of the deflection angle $\theta$ at which its centre of mass is deflected by the target.

Both distributions exhibit very similar features. For $\theta<2^{\circ}$, the oscillations are located at the same angles. A sort of Coulomb rainbow is observed at $2^{\circ}$ in both cases, and the oscillatory 


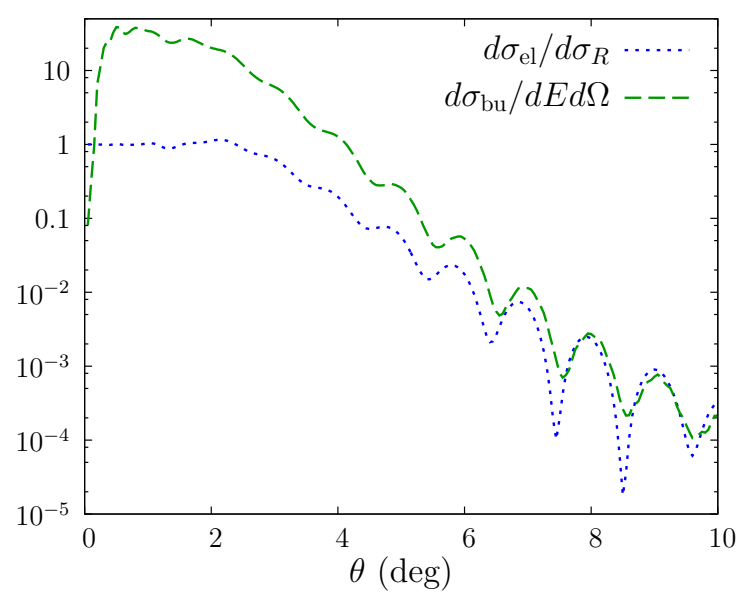

Figure 1: Angular distributions for elastic scattering and breakup of ${ }^{11} \mathrm{Be}$ impinging on $\mathrm{Pb}$ at $69 \mathrm{AMeV}$. The former is plotted as a ratio to the Rutherford cross section. The latter corresponds to a ${ }^{10} \mathrm{Be}-\mathrm{n}$ relative energy $E=0.3 \mathrm{MeV}$ and is expressed in $\mathrm{b} \mathrm{sr}^{-1} \mathrm{MeV}^{-1}$.

patterns at larger angles are very similar in location and magnitude. This suggests that the projectile is deflected by the target in the same way whether it remains bound or is broken up [U]]. This peculiarity can be easily explained within the Recoil Excitation and Breakup (REB) model [Ш2]].

\section{Recoil Excitation and Breakup model}

The REB model is based on two assumptions [ㅁ] ]. First it assumes an adiabatic treatment of the excitation of the projectile, i.e. the projectile excitation energy is neglected compared to the beam energy. Second, the neutron-target interaction is neglected. This model thus assumes the excitation of the projectile—or its breakup — to be due to the recoil of the core, hence its name.

With these two approximations, the REB provides an elegant and simple expression of the elastic scattering cross section of the projectile by the target

$$
\frac{d \sigma_{\mathrm{el}}}{d \Omega}=\left|F_{0,0}\right|^{2}\left(\frac{d \sigma}{d \Omega}\right)_{\mathrm{pt}}
$$

where $(d \sigma / d \Omega)_{\mathrm{pt}}$ is the elastic scattering for a pointlike projectile and $F_{0,0}$ is a form factor accounting for the extension of the halo

$$
F_{0,0}=\int\left|\Phi_{0}(r)\right|^{2} e^{i \vec{Q} \cdot \vec{r}} d \vec{r}
$$

with $\Phi_{0}$ the neutron-core wave function and

$$
\vec{Q}=\frac{m_{\mathrm{n}}}{m_{c}+m_{\mathrm{n}}}\left(\vec{K}-\vec{K}^{\prime}\right)
$$

is a fraction of the momentum exchanged in the elastic-scattering process $\left(m_{\mathrm{n}}\right.$ being the mass of the halo neutron and $m_{c}$ that of the core of the nucleus). 
Note that Eq. (1) is reminiscent of the first order of the Born series. However no such expansion has been performed here: the REB includes all orders of the scattering amplitude. It also includes the breakup channel and an expression similar to Eq. (B.D) can be derived for the breakup angular distribution

$$
\frac{d^{2} \sigma_{\mathrm{bu}}}{d E d \Omega}=\left|F_{E, 0}\right|^{2}\left(\frac{d \sigma}{d \Omega}\right)_{\mathrm{pt}}
$$

where the form factor now reads

$$
\left|F_{E, 0}\right|^{2}=\sum_{l j m}\left|\int \Phi_{l j m}(E, r) \Phi_{0}(r) e^{i \vec{Q} \cdot \vec{r}} d \vec{r}\right|^{2}
$$

with $\Phi_{l j m}(E)$ the core-neutron wave function in partial wave $l j m$ at energy $E$ in the continuum.

Within the REB assumptions, angular distributions (B.]) and (B.4) can be factorised as the product of a reaction part $(d \sigma / d \Omega)_{\mathrm{pt}}$ and a structure part $\left(\left|F_{0,0}\right|^{2}\right.$ and $\left|F_{E, 0}\right|^{2}$, respectively). The fact that the former is identical in both expressions explains why both distributions are so similar in Fig. 四.

\section{Ratio technique}

\subsection{Ratio idea}

The factorisations (B.D) and (B.4) also suggest that taking the ratio of these angular distributions removes most of the reaction dependence and hence provides an observable that enhances the sensitivity of the measurements to the projectile structure. It corresponds, within the REB assumptions, to the ratio of the form factors (B.5) and (B.2.

$$
\frac{d^{2} \sigma_{\mathrm{bu}} / d E d \Omega}{d \sigma_{\mathrm{el}} / d \Omega}=\frac{\left|F_{E, 0}\right|^{2}}{\left|F_{0,0}\right|^{2}}
$$

Besides its theoretical advantages, observable (4.]), being the ratio of two cross sections, does not require the normalisation of these cross sections, if both processes are measured within the same experimental setup.

\subsection{Testing the idea}

To test the validity of the ratio technique, we perform reaction calculations within the Dynamical Eikonal Approximation (DEA) [[13], [4]]. This model reproduces fairly well various experimental data [14] and is in excellent agreement with the Continuum Discretised Coupled Channel approach for reactions involving one-neutron halo nuclei at intermediate energy, i.e. at about $70 \mathrm{AMeV}$ or above [15]]. Since it does not rely on the REB assumptions (see Sec. [3), it is very well suited to test theoretically this ratio technique. We thus perform DEA calculations for ${ }^{11} \mathrm{Be}$ impinging on $\mathrm{Pb}$ at $69 \mathrm{AMeV}$, which correspond to the conditions of the RIKEN experiment of Ref. [䧃]. We use the inputs $\left({ }^{10} \mathrm{Be}-\mathrm{n}\right.$ interaction, optical potentials etc. $)$ and numerical parameters detailed in Ref. [14]]. 


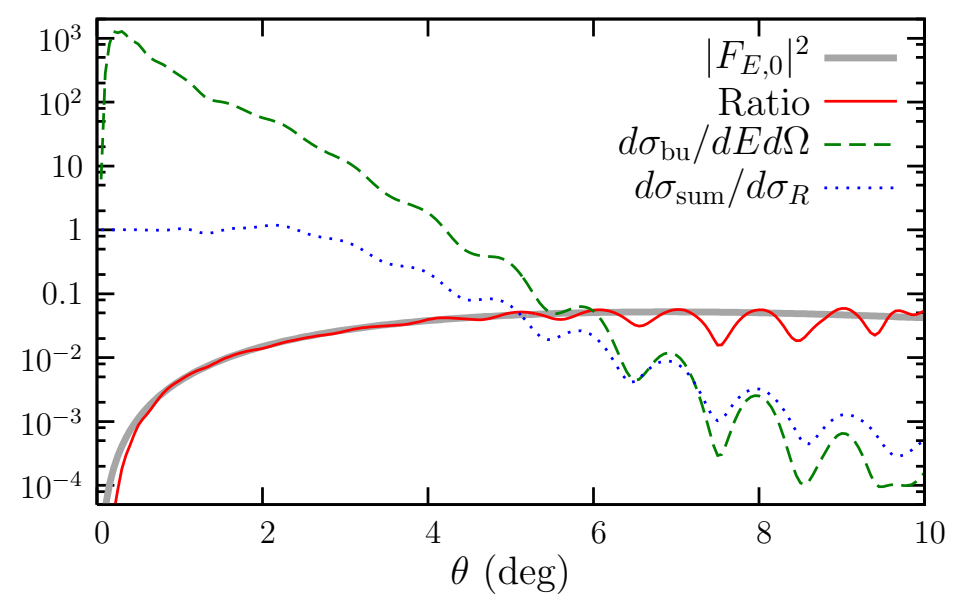

Figure 2: Ratio of angular distributions (4.3]) computed within DEA for ${ }^{11} \mathrm{Be}$ impinging on $\mathrm{Pb}$ at $69 \mathrm{AMeV}$ [ए]]. It is in excellent agreement with the REB prediction (B.5). The breakup angular distribution corresponding to a ${ }^{10} \mathrm{Be}-\mathrm{n}$ relative energy $E=0.1 \mathrm{MeV}$ is given in $\mathrm{b} \mathrm{sr}^{-1} \mathrm{MeV}^{-1}$ and the summed cross section (4.2) is plotted as a ratio to Rutherford.

Following the factorisations (B.D) and (B.4), the ratio of any linear combinations of angular distributions should be independent of the reaction process and give a ratio of form factors similar to (B.2) and (B.5). We have found optimal to consider the ratio of the angular distribution for breakup at one ${ }^{10} \mathrm{Be}$-n energy $E$ to the sum of the angular distributions for elastic and inelastic scattering and for breakup at all ${ }^{10} \mathrm{Be}-\mathrm{n}$ energies [ए]]

$$
\frac{d \sigma_{\mathrm{sum}}}{d \Omega}=\frac{d \sigma_{\mathrm{el}}}{d \Omega}+\frac{d \sigma_{\mathrm{inel}}}{d \Omega}+\int \frac{d^{2} \sigma_{\mathrm{bu}}}{d E d \Omega} d E
$$

The REB predicts this ratio to be equal to

$$
\frac{d^{2} \sigma_{\mathrm{bu}} / d E d \Omega}{d \sigma_{\mathrm{sum}} / d \Omega}=\left|F_{E, 0}\right|^{2}
$$

Fig. $\square$ displays the results of these calculations. As suggested in Secs. $\square$ and $\$$, the summed angular distribution (blue dotted line) and that for breakup (green dashed line) are very similar. As expected, their ratio (red solid line) removes most of the angular dependence. It varies smoothly with $\theta$ and is in excellent agreement with the REB prediction (4.3) (thick grey line). This confirms that the ratio observable probes the structure of the projectile with little dependence on the reaction mechanism.

\section{3 (In)Sensitivity to projectile-target interaction}

To confirm the independence of the ratio (4.3) of the reaction mechanism, we repeat the DEA calculations for a carbon target at $67 \mathrm{AMeV}$. Being nuclear dominated, this reaction mechanism is very different from that with a lead target. In Fig. [1], the ratios for the $\mathrm{Pb}$ (red solid line) and $\mathrm{C}$ (green dashed line) targets are compared to each other and with the REB prediction (thick grey line). Because the angle depends on the target choice and on the beam energy, these observables are plotted as a function of the modulus of $\vec{Q}(B .3)$ to ease the comparison. 


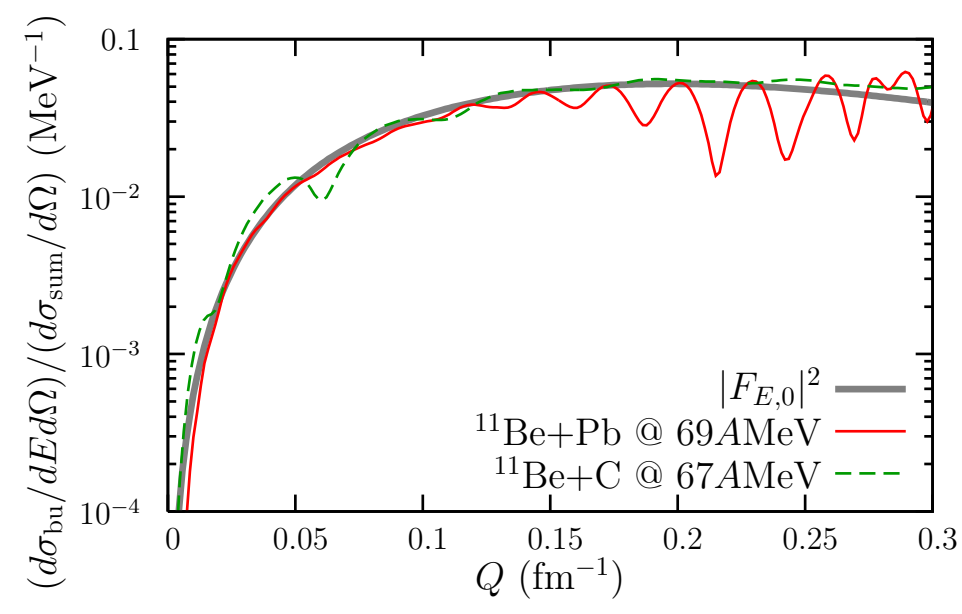

Figure 3: Sensitivity of the ratio technique to the reaction process. Ratio of angular distributions (4.3) computed within DEA for ${ }^{11} \mathrm{Be}$ impinging on $\mathrm{Pb}$ at $69 \mathrm{AMeV}$ and $\mathrm{C}$ at $67 \mathrm{AMeV}$ [山]]. They agree with each other and with the REB prediction (B.S).

The results are very similar for Coulomb- and nuclear-dominated collisions, confirming that the ratio (4.3]) is nearly insensitive to the reaction process. It seems thus very well suited to study the structure of halo nuclei.

To analyse the effect of the neutron-target interaction (neglected in the REB), we repeat the calculations on $\mathrm{Pb}$ without that interaction in the DEA. The results are plotted in Fig. 困 (black dash-dotted lines) and compared to the full calculations (coloured lines).

We observe that when the neutron-target interaction is switched off the large-angle behaviour of the ratio is smoother. As already noted by Johnson, Al-Khalili and Tostevin [ए2], the neutrontarget interaction shifts the oscillatory pattern at the large angles of the angular distribution. This

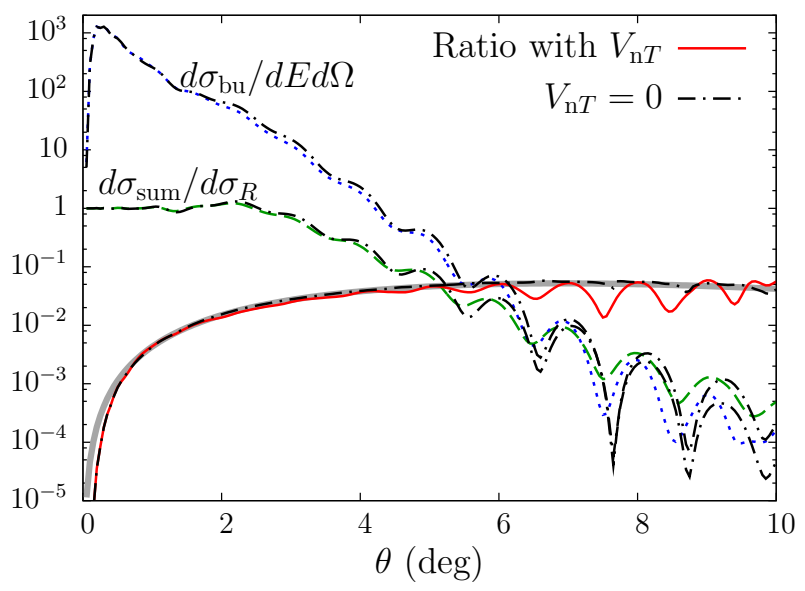

Figure 4: Influence of the neutron-target interaction $V_{\mathrm{n} T}$ on the ratio. DEA calculations for ${ }^{11} \mathrm{Be}$ impinging on $\mathrm{Pb}$ at $69 \mathrm{AMeV}$ are compared with and without $V_{\mathrm{n} T}$. 
shift is actually energy dependent, so it is slightly different for elastic scattering, inelastic scattering and breakup. Therefore without neutron-target interaction, both angular distributions exhibit exactly the same oscillatory pattern, and hence their ratio is very smooth, even at large angles. On the contrary, the angular distributions of the full calculations are slightly shifted from one another, explaining the remaining oscillations at large angles. Since the neutron-nucleus optical potential is well parametrised [16, [7], this is a minor problem of the technique.

\subsection{Sensitivity to the projectile description}

Knowing that the ratio (4.3) is nearly independent of the reaction mechanism, we now turn to the information that can be inferred from it about the projectile structure. Fig. [ (left) illustrates the sensitivity of the ratio to the binding energy of the valence neutron. Three DEA calculations are depicted for a ${ }^{11} \mathrm{Be}$-like projectile impinging on a lead target at $69 \mathrm{AMeV}$. The only difference between these calculations is the one-neutron separation energy of the projectile. The depth of the potential in the $s_{1 / 2}$ partial wave is modified to obtain different binding energies: $0.5 \mathrm{MeV}$ (the experimental value for ${ }^{11} \mathrm{Be}$; red solid line), $50 \mathrm{keV}$ (green dashed line) and $5 \mathrm{MeV}$ (blue dotted line). Each calculation is compared to its REB prediction (thick grey lines).

The ratio is very sensitive to the binding energy of the projectile. It varies both in shape and magnitude with the neutron separation energy. The DEA calculations are in excellent agreement with the REB prediction but for the deeply-bound system (binding energy of $5 \mathrm{MeV}$ ). In that case, the DEA ratio significantly underestimates the REB prediction at forward angles. This may be linked to the adiabatic approximation performed in the REB, which may no longer be valid for deeply-bound nuclei.

Fig. 1 (right) shows the sensitivity of the ratio to the partial wave of the valence neutron. Here also three DEA calculations are performed for ${ }^{11} \mathrm{Be}$-like projectiles impinging on $\mathrm{Pb}$ at $69 \mathrm{AMeV}$. In all cases the one-neutron separation energy is fitted to the experimental one $(0.5 \mathrm{MeV})$, but the neutron is bound in different partial waves to the ${ }^{10} \mathrm{Be}$ core: $1 s_{1 / 2}$ (red solid line), $0 p_{1 / 2}$ (green dashed line) and $0 d_{5 / 2}$ (blue dotted line). Here again a significant sensitivity to the projectile structure is observed in both shape and magnitude.
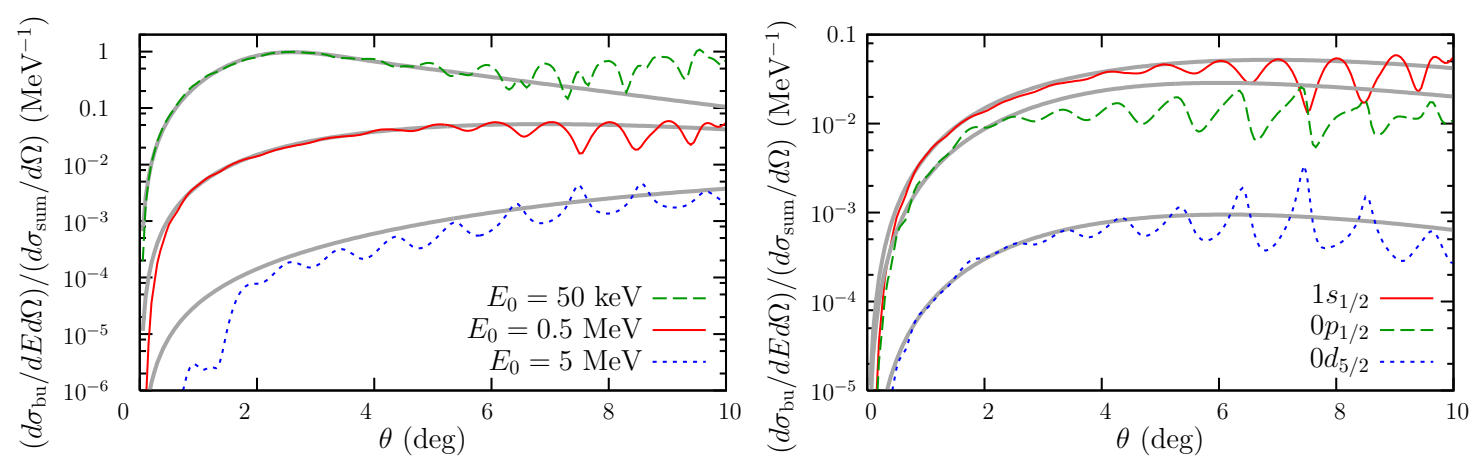

Figure 5: Sensitivity of the ratio (4.3]) to the projectile internal structure. DEA calculations are performed for ${ }^{11} \mathrm{Be}$-like projectiles impinging on $\mathrm{Pb}$ at $69 \mathrm{AMeV}$. The projectiles differ only by the neutron separation energy in the $1 s_{1 / 2}$ orbital (left) or by the partial wave in which the neutron is bound to ${ }^{10} \mathrm{Be}$ (right). 
We have also analysed the sensitivity of the ratio to the radial wave function by changing the geometry of the binding potential [ए0]. The sensitivity to the radial wave function is not as spectacular as observed in Fig. 5. The forward angle part of the ratio scales with the square of the Asymptotic Normalisation Constant (ANC) of the bound-state wave function (below $2^{\circ}$ at $69 \mathrm{AMeV}$ on $\mathrm{Pb}$ ). Interestingly, the ratio probes also the interior of the wave function at larger angles. Therefore, by removing the sensitivity to the reaction mechanism, the ratio gains sensitivity to parts of the wave functions that are inaccessible to most of the reaction observables. Usual breakup observables indeed probe only the asymptotics of the wave function [एष]].

\section{Summary}

The study of exotic nuclear structures is usually performed through reactions. In order to extract valuable information from experimental data, an accurate model of reaction coupled to a realistic description of the projectile is required. Alternatively, one can look for observables that are less dependent on the reaction process. In this work, we explore the ratio technique [ए0]]. This observable consists of the the ratio of two angular distributions and is predicted to be insensitive to the reaction mechanism. Using the DEA, we have tested this REB prediction and found that the ratio (4.3) removes most of the dependence on the reaction process for loosely-bound nuclei.

The ratio is also shown to be highly sensitive — in both shape and magnitude - to the binding energy and the partial-wave configuration of the valence neutrons. To a lesser extend, it is also sensitive to the radial wave function of the halo [ए]] . Of course this idea should be tested on actual experimental data to evaluate its applicability. Nevertheless, the present study shows that the ratio technique is quite promising.

The formalism used here assumes a single configuration of the wave function, i.e. a spectroscopic factor equals to one. In the future, we plan to extend this analysis to more realistic descriptions of the projectile that include various configurations. Open questions remain: is it the possible to extend the ratio technique to two-neutron halo nuclei? what about proton halos? If these extensions were possible, the ratio would open a new era in the study of structure of exotic nuclei.

\section{Acknowledgments}

This work was supported by the National Science Foundation grant PHY-0800026 and the Department of Energy under contract DE-FG52-08NA28552 and DE-SC0004087. It is also part of the Belgian Research Initiative on eXotic nuclei (BriX), program P6/23 on inter-university attraction poles of the Belgian Federal Science Policy Office. R.C.J. is supported by the United Kingdom Science and Technology Facilities Council under Grant No. ST/F012012. 


\section{References}

[1] I. Tanihata, H. Hamagaki, O. Hashimoto, S. Nagamiya, Y. Shida, N. Yoshikawa, O. Yamakawa, K. Sugimoto, T. Kobayashi, D.E. Greiner, N. Takahashi, Y. Nojiri, Phys. Lett. B160, 380 (1985)

[2] I. Tanihata, J. Phys. G 22, 157 (1996)

[3] P.G. Hansen, B. Jonson, Europhys. Lett. 4, 409 (1987)

[4] N. Fukuda, T. Nakamura, N. Aoi, N. Imai, M. Ishihara, T. Kobayashi, H. Iwasaki, T. Kubo, A. Mengoni, M. Notani, H. Otsu, H. Sakurai, S. Shimoura, T. Teranishi, Y.X. Watanabe, K. Yoneda, Phys. Rev. C 70, 054606 (2004)

[5] T. Nakamura, N. Fukuda, N. Aoi, N. Imai, M. Ishihara, H. Iwasaki, T. Kobayashi, T. Kubo, A. Mengoni, T. Motobayashi, M. Notani, H. Otsu, H. Sakurai, S. Shimoura, T. Teranishi, Y.X. Watanabe, K. Yoneda, Phys. Rev. C 79, 035805 (2009)

[6] T. Nakamura, N. Kobayashi, Y. Kondo, Y. Satou, N. Aoi, H. Baba, S. Deguchi, N. Fukuda, J. Gibelin, N. Inabe, M. Ishihara, D. Kameda, Y. Kawada, T. Kubo, K. Kusaka, A. Mengoni, T. Motobayashi, T. Ohnishi, M. Ohtake, N.A. Orr, H. Otsu, T. Otsuka, A. Saito, H. Sakurai, S. Shimoura, T. Sumikama, H. Takeda, Phys. Rev. Lett. 103, 262501 (2009)

[7] A. Di Pietro, G. Randisi, V. Scuderi, L. Acosta, F. Amorini, M.J.G. Borge, P. Figuera, M. Fisichella, L.M. Fraile, J. Gomez-Camacho, H. Jeppesen, M. Lattuada, I. Martel, M. Milin, A. Musumarra, M. Papa, M.G. Pellegriti, F. Perez-Bernal, R. Raabe, F. Rizzo, D. Santonocito, G. Scalia, O. Tengblad, D. Torresi, A.M. Vidal, D. Voulot, F. Wenander, M. Zadro, Phys. Rev. Lett. 105, 022701 (2010)

[8] J. Al-Khalili, F.M. Nunes, J. Phys. G 29, R89 (2003)

[9] D. Baye, P. Capel, in Clusters in Nuclei, Vol. 2, Lecture Notes in Physics 848, ed. by C. Beck (Springer, Heidelberg, 2012)

[10] P. Capel, R. Johnson, F. Nunes, Phys. Lett. B705, 112 (2011)

[11] P. Capel, M. Hussein, D. Baye, Phys. Lett. B693, 448 (2010)

[12] R.C. Johnson, J.S. Al-Khalili, J.A. Tostevin, Phys. Rev. Lett. 79, 2771 (1997)

[13] D. Baye, P. Capel, G. Goldstein, Phys. Rev. Lett. 95, 082502 (2005)

[14] G. Goldstein, D. Baye, P. Capel, Phys. Rev. C 73, 024602 (2006)

[15] P. Capel, H. Esbensen, F.M. Nunes, Phys. Rev. C 85, 044604 (2012)

[16] F.D. Becchetti, Jr., G.W. Greenlees, Phys. Rev. 182, 1190 (1969)

[17] A.J. Koning, J.P. Delaroche, Nucl. Phys. A713, 231 (2003)

[18] P. Capel, F.M. Nunes, Phys. Rev. C 75, 054609 (2007) 